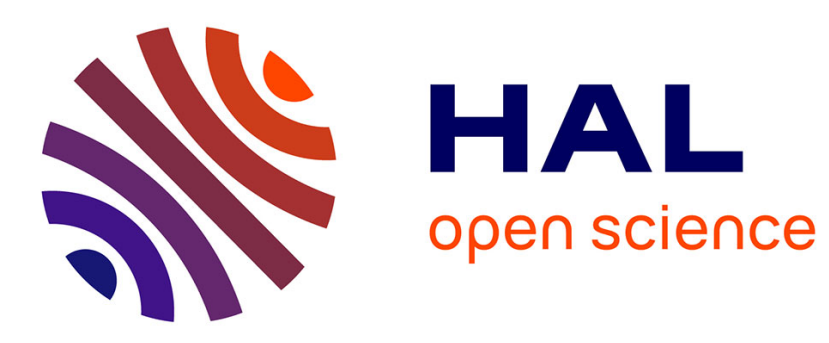

\title{
La prévention mondiale de la COVID 19: un subalternisme hégémonique des subalternisé.es
}

\author{
Jacqueline Descarpentries
}

\section{To cite this version:}

Jacqueline Descarpentries. La prévention mondiale de la COVID 19: un subalternisme hégémonique des subalternisé.es. Recherches \& éducations, 2020, 10.4000/rechercheseducations.9743 . hal-03286390

\section{HAL Id: hal-03286390 \\ https://hal.science/hal-03286390}

Submitted on 18 Aug 2021

HAL is a multi-disciplinary open access archive for the deposit and dissemination of scientific research documents, whether they are published or not. The documents may come from teaching and research institutions in France or abroad, or from public or private research centers.
L'archive ouverte pluridisciplinaire HAL, est destinée au dépôt et à la diffusion de documents scientifiques de niveau recherche, publiés ou non, émanant des établissements d'enseignement et de recherche français ou étrangers, des laboratoires publics ou privés. 


\title{
La prévention mondiale de la COVID 19: un subalternisme hégémonique des subalternisé.es
}

\author{
Jacqueline Descarpentries
}

\section{Introduction}

Une interrogation constante avec la critique foucaldienne de la prévention des dispositifs de prévention de l'OMS met ici en exergue les tensions majeures de la construction sociale et politique des dispositifs de prévention pensés en soi comme la normalité et l'universel. Une analyse critique de l'usage social et politique des résultats de la recherche épidémiologique(Latour,1997), pharmaceutique et de santé publique dans la pandémie de la COVID 19 définit en effet, non seulement la prétention universaliste mondiale de gouverner la santé individuelle et communautaire des populations, mais aussi la prétention à renvoyer des Etats à un subalternisme (Amselle, 2008) de l'hégémonie (Gramsci, 1948) de la politique de la vie (Fassin , 2008) par une politique de santé publique onusienne de tri et de thanatocracie (Anselme, 2020). La pandémie a en effet révélé non seulement l'usage social et politique des résultats de la recherche, mais aussi le pouvoir hégémonique d'une essentialisation stratégique (Zpivak, 2013) des résultats de recherches en épidémiologiques qui ont défini la subalternisation des populations subalternes pour mieux justifier un ordre colonial mondial de santé publique coloniale.

Par un examen soutenu et instructif de ses prémisses, l'article présente une réflexion sur le dispositif de prévention de la COVID 19 qui, par la vision linéaire marxiste et hégélienne de l'histoire des idées, postule comment toutes autres cultures de santé onusienne ne sont que des formes primitives ; mais aussi comment la mathématisation de la vie et du vivant exerce une hégémonie par l'usage social et politique de la vérité dans la recherche en santé publique. Un subalternisme hégémonique s'est ainsi imposé comme un nouvel ordre mondial par la relégation des politiques locales de prévention et des comportements de protection de santé individuelle et collective par un dispositive de l'essentialisation stratégique des savoirs pour mettre en œuvre un politique mondiale de santé publique coloniale.

\section{La socialisation de la recherche en santé publique}

Pour Bruno Latour (2020)1, dans ses premiers travaux et aujourd'hui dans ses engagements " épistémologiques » fondamentaux, la sociologie empirique des sciences ne peut pas encore " cerner le virus, socialement, politiquement, collectivement " même si "l'activité scientifique ne peut être séparée des cadres sociaux au sein desquels elle se réalise, car toute " vérité "scientifique est une construction, ce qui signifie qu'elle ne saurait découler de la démonstration et de la preuve publique ». Bruno Latour avait précisé par ailleurs, la classique distinction entre la logique de la justification et la logique de la découverte non pertinente, puisque le conceptuel, le matériel et l'instrumental ne peuvent être séparés du social et du politique (Latour, 2006). II rappelait aussi I' imposture à prendre la théorie pour un programme d'actions alors qu'elle est un corpus cohérent d'arguments explicatifs (Latour, 1989). Une théorie explicative n'est pas une compréhension de l'action. L'argumentaire théorique sur une recherche opératoire de la pragmatique pour améliorer la pratique est insuffisant, car l'opératoire n'est pas opérationnel et la recherche est d'abord démonstrative, 
explicative et spéculative : " La pragmatique n'est pas la praxéologie qui se donne pour volonté obsessionnelle « d'optimiser les pratiques » (Vial, 1997).

La praxistique donne ainsi une autre conception aux savoirs, elle donne un statut « du nécessaire à la théorie » en lui reconnaissant son caractère général et explicatif. C'est ainsi que dans l'analyse critique du dispositif de la pandémie de la COVID 19 tel que géré par I' OMS, a fait émerger un usage social de la construction de la recherche en santé publique et un usage social et politique des résultats de des recherches dans la mise en place des dispositifs de prévention liés à une négociation du sens de ces mêmes résultats de la recherche dans leurs usages dans les décisions politiques de prévention par les Etats (Descarpentries, 2018).

\section{Une normalité et une universalité au service des politiques de santé publique}

En effet, le dispositif de la pandémie onusienne est mondial et les catégories

d'objectivité scientifique utilisées pour définir les politiques de prévention dans les Etats n'en sont pas moins historiquement situées. Elles sont aussi devenues des catégories locales de sens mondialisé (De Sousa Santos, 2016) en raison de leur universalité doxique scientifique liée à la circulation de la norme de la vérité scientifique. Dès lors, si on admet qu' une vérité scientifique a fait l'objet d'une universalisation dans le social (Balibar, 2016), on comprend comment les modèles de prévention mis en place, dès l'apparition de la maladie en Chine, ont été pris comme modèle mondial de prévention, selon les savoirs scientifiques qui circulent en raison de leur universalité par l'évidence contraire selon laquelle ils sont décrits comme universels précisément parce qu'ils circulent comme norme, renforcée par des enjeux politiques implicites.

De plus, force est de constater que la pandémie a réveillé les débats légitimes entre nature et culture, car comme Bruno Latour le précise dès le 14 mai 2020 : « Le virus n'a rien à voir avec la "nature". Quand on voit la diversité incroyable des réactions au virus, que ce soient les corps individuels, les corps sociaux, les corps nationaux, on s'aperçoit que l'image d'un événement de la nature qui tomberait de l'extérieur et uniformément sur les pauvres humains n'a rigoureusement aucune espèce de sens ${ }^{2}$. Si il existe une réalité naturelle indépendante du virus et il est aussi inscrit dans une réalité sociale de recherche fondée par des activités cognitives liées à des controverses, mais aussi à critères externes, comme, tout particulièrement le poids des logiques des chercheurs entre eux, du politique, de l'industrie pharmaceutique pour les traitements et de l'industrie marchande pour les masques et les tests dépendante des économies locales, nationales et internationales, voire technologiques (Latour, 2017) Héritière de Thomas Kuhn et, surtout, de Paul Feyerabend, si la sociologie des sciences contemporaine attribue une place prépondérante au sens et aux preuves qui seraient socialement négociés entre scientifiques qui s s'expliquent, notamment, par le fait que les théories sont " sous-déterminées " par les faits. Le refus de la distinction entre une preuve expérimentale et une conviction mène toutefois tout droit au relativisme épistémologique (Landry 1935) où, les chercheurs à l'international prennent souvent à leur compte les schèmes occidentaux sans questionner la naissance de la pensée qui est toujours inscrite dans un contexte donné (y compris pour la pensée universelle).

Ainsi, en identifiant la vérité au consensus, il est douteux que la sociologie des sciences échappe au paradoxe de la composition lié au fait que ce qui est vrai dans l'instant ou dans le court terme peut devenir faux dans le long terme (Boghossian, 2009). Et ce, même si on ne peut pas déterminer la vérité tout de suite, ceci n'autorise pas à conclure qu'il n'existe pas de vérité, pas plus qu'il existe une vérité universelle. Si par ailleurs, on ne 
peut sous-estimer l'apport de Michel Foucault, avec l'archéologie des savoirs ; c'est-à-dire les conditions de production des discours sur la sexualité, la folie ou la prison, comme il l'a fait, il n'est effectivement pas possible de nier les conditions de production des savoirs et encore moins les conditions épistémologiques qui déterminent les conditions de la vérité. Dans un entretien de 1977, Foucault affirme en effet que "la "vérité" est liée circulairement à des systèmes de pouvoir qui la produisent et la soutiennent, et à des effets de pouvoir qu'elle induit et qui la reconduisent.» ${ }^{3}$. Dès lors, ce ne sont pas les faits qui nous contraignent mais le "régime de vérité " de la société à laquelle nous appartenons. Ce raisonnement reste ainsi un idéal-typique du constructivisme de la justification locale et contingente pour les chercheur.es. Dans l'« épistémologie » de Foucault, il n'existe donc aucune place pour la distinction entre être vrai et être tenu pour vrai. Il est pourtant essentiel de ne pas confondre, comme le souligne Jacques Bouveresse, le caractère historiquement déterminé des moyens dont nous disposons pour décider si une proposition est vraie ou fausse avec "la vérité ou la fausseté de la proposition, qui peut très bien être déterminée sans que nous y soyons pour quelque chose »(2001). Dans la pandémie de la COVID 19, force est alors de reconnaitre que les scientifiques ont fini par s'accorder, malgré les nombreuses controverses, pour décider quels résultats de recherches possédaient des raisons de les croire ou de les tenir pour vrais et donc pour norme. Une telle position des scientifiques a permis non seulement à l' OMS de déterminer une politique mondiale de gestion de la pandémie, mais aussi aux Etats de construire leurs propres politiques de santé publique selon le principe de subsidiarité. Force a donc été de constater la prétention de l' OMS à développer une santé globale et mondiale qui vise à gouverner le monde au nom de la raison universelle gouvernée par la mathématisation de la vie et du vivant, quelles que soient les aires géographiques.

\section{Entre hégémonie et subalternisation}

Trois mois après le début officiel de pandémie en Europe, des conséquences fort dommageables, économiques, sociales et individuelles ont émergé par l'usage de ces résultats de recherches par les politiques. D'une part, on peut nommer la mise en place 'un dispositif panoptique de confinement historique basé sur un régime de contrôle visant la normation des comportements des populations quelles que soient les aires géographiques, les systèmes politiques, économiques, d'accès aux soins, à l'eau ou à l'alimentation. Ce régime de contrôle disciplinaire des populations a en effet été rendu possible par un panoptique étatique de privation des libertés (Foucault, 1975) et de mise en place de différentes applications de traçages, soumettant chacun.e à une autosurveillance au domicile et au traçage des comportements individuels lors des sorties autorisées ou non selon les Etats. D'autre part, la mise en œuvre d'un biopouvoir et d'une biopolitique d'une santé publique mondiale normative a par ailleurs été possible par différents dispositifs : l'universalisation d'un modèle de prévention rendue hégémonique quelles que soient les aires géographiques (Gramsi,1948) et par une interprétation locale et contingente par les politiques des Etats des résultats de la recherche épidémiologique et de santé publique. Une heuristique de la peur (Jonas, 1979) a aussi entretenue la peur par un usage médiatico-politique des informations médicales mondiales rendues publiquement incertaines par un discours politique d'informations contradictoires favorable à la naissance de tout dispositif totalitaire (Arendt, 1951) savamment entretenu par la comptabilisation médiatisée du nombre de morts pour les pays les plus riches, allant vers des sanctions financières, policières, voire aussi par des délations. 
Force est par ailleurs de constater que la prévention de la COVID 19 a révélé l'extraordinaire pouvoir de normation des Etats dans le monde entier et l'extraordinaire soumission volontaire ou involontaire (Pinceau-Charlot, 2007) des citoyens confrontés aux risques de mort éminente et à la fin de la croyance dans le progrès. La prévention de la COVID 19 a ainsi mis en évidence les profondes inégalités de nos sociétés qu'elles soient des systèmes de santé, d'accès à la prévention et aux soins, à l'eau et à l'alimentation. Elle a révélé par ailleurs une crise profonde du néolibéralisme mondial où s'exerce un darwinisme biologique (que les plus forts survivent) et un darwinisme social (celui qui veut avancer se battra ...les autres tomberont).

En France, elle a ainsi soumis les invisibles (Gramsi,1948) à la sauvegarde du bien-être de la population confinées (comme les éboueurs, les camionneur.es, les femmes et hommes de ménages, les vendeurs et vendeuses dans les magasins d'alimentation...). Elle a exercé une marginalisation des enfants les plus vulnérables, elle a soumis des femmes à la violence. Elle a révélé les disparités de genre domestique et au travail, elle a su dé-confiner les sans-abris mis à l'abris sans éthique, ni morale, elle a enterré nos morts sans respect des traditions funéraires. Elle a mis sur les routes en Inde des hordes de populations qui ont fui la famine et les répressions policières, elle a laissé des corps morts dans des sacs plastiques au milieu des rues en Equateur. Elle crée la famine au Pérou. Elle a n'a pas empêcher la contamination des peuples des forêts en Amazonie... (Aguilar et all, 2020)

Le dispositif de prévention du COVID montre aussi combien la prévention n'est pas une priorité des politiques de santé puisqu'il est surprenant qu'au XXIème siècle la recherche en santé publique promeut toujours les mêmes gestes barrières et de confinements connues depuis les pandémies dans le monde (Sidéris, 2020) au risque de toutes les dérives humaines, sociales et économiques du monde contemporain et de la mondialisation. Il est tout aussi questionnant de constater comment l' OMS met en place un dispositif mondial de prévention en voulant exercer une gouvernance de la vie et du vivant, en expérimentant des dispositifs de contrôles pluriels à partir d'un usage social et politique de la recherche construite comme norme des politiques de santé publique des Etats pour une santé mondiale et globale.

La prévention de la COVID 19 par son dispositif d'un régime de normalisation disciplinaire (Foucault, 1975) a tenté de rendre les gens et les actes conformes au modèle hégémonique de la norme épidémiologique en santé publique mondiale issue de la seule mathématisation de la vie, du vivant et de l'environnement, dont le monde politique a fait usage, en France notamment, pour rester au chevet du nombre de lits dans les hôpitaux dont les personnels sont maltraités par les coupes budgétaires depuis les dernières crises économiques. La prévention de la COVID 19 a toutefois montré dans de nombreux pays, des dispositifs de soutien et de solidarités pour faire face à la pandémie pendant le confinement, comme dispositif d'expériences en marge à la biopolitique qui traduit non seulement un dispositif de résistance de territoires par une insoumission à la technique de normalisation des corps, de la psyché et de l'altérité, mais aussi comme l'exercice de pouvoir de religions en sauvegarde des communautés (Karray, 2020). L'expérience de ces solidarités pour sauvegarder la santé des populations, quels que soient les contextes marque ainsi des configurations politiques, sociales et morales différentes dans laquelle la vie physique et psychique s'est imposée comme valeur supérieure et la raison humanitaire comme idéal éthique.

Autrement dit, la pandémie de la COVID 19 nous apprend que pour d'aucun les politiques publiques, par un discours performatif de protection des populations ont mis en place une politique de privation des libertés par la peur, le contrôle, le traçage, voire les violences policières. Pour d'autres, les valeurs de solidarité, de tolérance ou de liberté sont restées la valeur instrumentale, voire une valeur substantielle de la vie. On pourrait 
même craindre que l'abandon des valeurs éthiques de la vie dans la recherche finisse par être un problème pour la démocratie. D'autant que l'on a pu observer comment des Etats ont fait un usage politique de la pandémie pour renforcer leur politique interne que ce soit en Europe, au Brésil ou aux Etats- Unis. On a pu aussi observer comment la colère gronde.

C'est ainsi que ce projet mondial colonial de contrôle de la santé publique mondiale et globale des populations, a fait émerger des crises économique, sociale, humaine, mais aussi une crise épistémologique majeure car les l'épistémologies de la cherche est la première source des inégalités (De Sousa Santos, 2016). La pandémie de la COVID 19 inséparable du projet de critique des savoirs hégémoniques de la rationalité du "Nord global ", auxquels s'ajoutent la politique sanitaire de la Chine et de ses orientations internes politiques a montré l'évolution des usages sociaux et politiques des résultats de la recherche en santé publique dans le contexte de la mondialisation des dispositifs de lutte contre la COVID 19. En fait, elle a notamment modifié la question générale de la capacité de lecture critique par les problèmes que soulèvent l'essentialisation des résultats de recherches pour justifier les pratiques d'une politique mondiale de santé publique coloniale.

\section{Une essentialisation stratégique des résultats de la recherche en santé publique}

En effet, toute étude de santé publique portant sur une collectivité fondée sur des

populations plurielles doit affronter le problème de l'essentialisme, d'abord en tant que celui-ci a été à l'œuvre et continue de l'être dans le portrait d'une collectivité brossé par les autres (scientifique ou autres), y compris dans l'interprétation des analyses biologiques, puis en tant que ce que l'on fait usage de ces savoirs pour décrire leur différance (Derrida, 1967). C'est-à-dire ce qui est fait des résultats de la recherche qui construit des catégorisations de sujets à risques hors normes : autrement dit des sujets subalternisées par l'hégémonie de la norme en santé publique : des communautés de subalternes définis par des catégorisations, non seulement de risques, mais discriminantes, parce que ségrégées par la mathématisation de la vie et du vivant, mais aussi, par tout dispositif de stigmatisations (pauvreté, genre, vulnérabilités sociales et d'accès aux soins, à l'eau et à l'alimentation), de disciplinarisation des corps et de la psyché par la ghettoïsation du confinement. On a ainsi pu observer une utilisation stratégique d'un essentialisme " positif " pour servir des objectifs politiques clairement définis par différents Etats dans le but d'instaurer une domination normative locale et contingente mais aussi universelle en appliquant les directives de santé publique mondiale et globale ${ }^{4}$.

En essentialisant les populations à protéger par le confinement et les populations subalternisées chargés de nourrir, de soigner et à trier les ordures, mais aussi les sans-abris, l'essentialisme a nourri la fixité des subalternes par un usage des stéréotypes des pauvres, des marginalisés, des indigents, des sans-domiciles-fixes accompagnés de leur invariabilité épidémiologique mathématique des facteurs de risques (diabète, obésité MCV...). En percevant les risques de contamination et les facteurs de risques associés une certaine catégorie essentielle d'individus à risques est par ailleurs apparue dans la propagation de la maladie, populations le plus souvent les plus précaires ${ }^{5}$. Les politiques publiques de santé publique ont ainsi trouvé dans ce type de synthèse et dans l'histoire des pandémies, la justification des interventions de prévention, mais aussi un dispositif de ghettoïsation (confinement) commode par le contrôle des populations. 
Le confinement s'est ainsi révélé comme un programme de redressement des corps et de la psyché au nom de la protection justifiée historiquement depuis le début des pandémies.

Par ailleurs, si comme l'explique Diana Fuss, l'essentialisme est le plus généralement interprété comme une croyance en la véritable, la vraie essence des choses, en l'existence de propriétés invariables et fixes qui définissent la " quiddité » d'une entité donnée [...]. Chose importante, l'essentialisme est, de façon caractéristique, défini par opposition à la différence [...]. L'opposition est utile dans la mesure où elle nous rappelle que c'est un système complexe de différences culturelles, sociales, psychiques et historiques qui situe et constitue le sujet, et non un ensemble d'essences humaines préexistantes. Mais l'articulation binaire entre essentialisme et différence peut aussi être réductrice, voire faire diversion, dans la mesure où elle nous autorise à ignorer ou à nier les différences réelles soulignées par l'essentialisme. (Fuss 1987); cette manière nuancée d'envisager l'essentialisme comme étant en lui-même caractérisé par la différence nous permet de comprendre que la différence et l'essentialisation des pratiques de recherches en santé publique peuvent agir comme les deux faces d'une même médaille des populations à risques subalternisées comme objet de recherche est employée pour déterminer des " bonnes pratiques " : les évidences base practices issus des catégorisations mathématiques - Leur usage social et politique permet alors de stéréotyper et caractériser des individus et des groupes au nom des principes d'éthique de la santé publique des populations pour des motivations politiques multiples et avec des conséquences multiples plus proches $\mathrm{du}$ colonialisme mondial pour un modèle unique de santé globale ou mondiale. Ces stéréotypes essentialistes peuvent alors être employés, et l'ont été, pour créer des hiérarchies entre populations pour imposer un modèle unique de prévention de la COVID 19 pour une politique de santé publique mondiale globale associée à la financiarisation de la santé publique et à l'exercice d'un régime de contrôle par les Big Data renforcé par différents dispositifs iatrogènes selon les pays : habitats trop petits, limitation des droits à l'accès aux soins, à l'eau et à l'alimentation, spoliation des terres qui supprime les sources alimentaires, pollution des eaux, pendant le confinement selon les pays, mise en place des inhumation indignes, soumission à la domination patriarcale de politiques coloniales de santé publique sur le corps des subalternisés (les femmes, les enfants, les handicapés, les vieux, les pauvres et les SDF...), domination policière et la famine entrainant des hordes de migration de populations.

On a également pu observer que la description essentialisée prétendument scientifique des populations à risques par la mathématisation a été employée pour justifier des programmes de développement de l'industrie pharmaceutique, ignorant les remèdes locaux. Enfin, avec les études épidémiologiques, il est impossible d'éviter les pièges du déterminisme biologique ou de la fixité du stéréotype en continuant d'employer l'essentialisme de manière consciente et réfléchie. Une forme $d^{\prime}$ '« essentialisme stratégique " s'est construit, tel que décrit par Spivak quand elle décrit l'usage tactique et délibéré de la typologie essentialiste : c'est-à-dire " un usage stratégique de l'essentialisme positiviste à des fins politiques clairement visibles " écrivait-elle en 1996. En nuançant l'usage que Spivak fait de l'essentialisme stratégique, force est de constater que même si la conception positiviste, n'est plus le dispositif essentiel de la mathématisation de la vie et du vivant, il n'en demeure pas moins qu'un darwinisme social et biologique qui a déjà été mentionné participe à la l'usage essentialiste des déterminants de santé et à la ghettoïsation des subalternes. 


\section{Conclusion provisoire}

Si l'essentialisme stratégique ne peut pas contrer l'effacement ou l'omission opéré par les travaux universitaires classiques de l'épidémiologie, le/la chercheur/e critique se doit alors de veiller à ne pas reproduire le mécanisme du discours essentialiste pour que ne produisent pas des catégorisations de types d'altérité purement symboliques qui seront ghettoïsées. Le concept d'essentialisme stratégique nous permet ainsi de rester vigilant.e dans la compréhension de la construction sociale et politique de la prévention en santé publique et de la volonté mondiale d'une santé publique globale coloniale. Même si les groupes à risques sont des populations subalternisées, il n'en demeure pas moins que ces catégories de pensées participent à l'identification de leur caractéristique qui fait figure de référence, de catégorisations et de justification d'interventions, telles une essence au point que les groupes s'auto décrivent à partir de ces critères essentialisés.

Même si ces catégories permettent aux populations subalternisées par la recherche et par l'usage de ces résultats de recherches, elles permettent aussi à ces groupes de se mobiliser. Le concept d'essentialisme stratégique soutient en effet que la fixation provisoire d'une essence dont on sait qu'elle n'est pas artificielle peut dans certains cas être stratégiquement utile. Autrement dit, l'essentialisation des résultats de la recherche en santé publique doit être approfondie autant sur le plan théorique que pour ses effets sur la pratique opérationnelle dans les programmes de prévention, car il tend à paralyser l'action, car toute action suppose la formation de collectifs, et les collectifs tendent à « essentialiser » leur identité ce qui pose la question des conditions pratiques d'émergence de l'action collective.

Dans un contexte marqué par la pandémie de la COVID 19, il faut reconnaître que la grille d'analyse de la prévention par l'essentialisme stratégique telle que pensée par Spivak apporte des éclairages nouveaux pour la recherche critique des dispositifs de prévention dans les politiques de santé publique. Si le virus a une cruelle pédagogie comme le précise De Sousa Santos (2020)6 la transformation sociale de la recherche et des pratiques opérationnelles des éducations critiques et coopératives dans le champ de la santé restent un des levier de la prévention émancipatrice qui est à opérer pour une promotion de la santé démocratique qui exige débat, dialogues, la diversité, par un examen critique soutenu et instructif des prémisses, des méthodes de la santé publique pour éviter les tensions majeures d'une colonisation de la santé globale et mondiale.

\section{BIBLIOGRAPHIE}

Aguilar Y. E. (2020). Capitalismo y Pandemia. Edition : Filosophia Libre.

Arendt H. (1951). Les origines du totalitarisme. Paris : Gallimard.

Bouveresse J. (2001). L'objectivité, la connaissance et le pouvoir in Didier Éribon

(dir.), L'Infréquentable Michel Foucault, Paris : EPEL.

Buekens P., Bréart G., Cot M., Leveque A., Massougbodji A., Rodriguez López M.H., (2011).

Global health : A globalized public health, in Revue d'Epidémiologie et de Santé Publique, 59, $\mathrm{n}^{\circ}$

2, 73-75. 
Charlot M, Pinçon-Charlot M. (2007). La sociologie de la bourgeoisie. Paris : La Découverte.

Derrida J. (1967). L'écriture et la différence. Paris : Editions de Minuit.

De Sousa Santos, B. (2016) Épistémologies du Sud. Mouvements citoyens et polémique sur la science, Paris : Desclée de Brouwer, coll. Solidarité et société,

De Sousa Santos B. (2020). La cruel pedagogia del virus. Lisbonne : Editions Clacso.

Descarpentries J. (2018). Pour une éducation à la santé durable, in revue Open Source Educations.

Fassin D. (2000). Entre politiques de la vie et politiques du vivant.

Pour une anthropologie de la santé". In Anthropologie et Sociétés, 24, 1, 2000, pp. 95-116. Québec

: département d'anthropologie, Université Laval.

Foucault M. (1975). Surveiller et Punir. Paris : Gallimard.

Foucault M. (1979). La naissance de la biopolitique, Cours au collège de France 78-79, Paris :

Gallimard.

Fuss D. (1989). Essentially Speaking: Feminism, Nature and Difference. New York: Editions du Rougtledge.

Karray A, Derivois D. (2020). Quelle résilience pour quels modèles de société, in The Conversation.

Jonas H. (1979). Le principe responsabilité. Paris : Les Champs, Essais.

\section{NOTES}

1. https://www.telos-eu.com/fr/societe/bruno-latour-le-virus-et-la-verite.html\#_ftn3

2. Libération, $14-5-2020$, p. 20

3. Michel Foucault, Dits et écrits, Gallimard, 1994-2001, p. 160. Un " régime de vérité " est constitué par un système épistémique (les règles de justification des énoncés) et par les dispositifs de pouvoir dans lesquels il s'inscrit.

4. https://www.em-consulte.com/en/article/285973

5. https://www.oxfamfrance.org/inegalites-et-justice-fiscale/covid-19-le-virus-des-inegalites/

\section{RÉSUMÉS}

La pandémie de la COVID 19 a fait l'objet de très nombreux articles en SHS et a été étudiée, par de nombreux auteurs critiques(Préciado, 2020), comme un dispositif, au sens foucaldien, de santé publique mondiale qui s'est imposé comme un dispositif de biopouvoir et de biopolitique quels que soient les pays. A travers, l'analyse critique de la construction des savoirs et de leur usage social et politique se lit un exercice de pouvoir dans la normation des corps et de la psyché par un dispositif panoptique de contrôle des populations; mais aussi comment s'est construite une essentialisation stratégique (Zpivak,2013) des savoirs de l'épidémiologie (des déterminants de santé et des facteurs de risque) par la subalternisation des populations subalternes pour favoriser une politique de santé publique mondiale globale (Buekens, 2011) coloniale centrée sur un régime de contrôle des populations. La pandémie de la COVID 2020 ne traduirait-elle pas ainsi une crise épistémologique majeure entre la recherche académique et les approches opérationnelles éthiques de la prévention? 
The COVID 19 pandemic has been the subject of numerous articles in HSS and has been studied, by many critical authors, as a device, in the Foucauldian sense, of global public health which has established itself as a device biopower and biopolitics whatever the country. Through this, the critical analysis of the construction of knowledge and its social and political use is effectively read an exercise of their power in the normalization of bodies and the psyche by a panoptic device of population control; but also how a strategic essentialization of knowledge of epidemiology (health determinants and risk factors) was built by a social and political use of ecological knowledge of subordinate populations (Zpivak, etc.) favorable to a global public health policy colonial system centered on a regime of control of populations. Does the COVID 2020 pandemic not reflect a major epistemological crisis between academic research and operational approaches to prevention?

\section{INDEX}

Keywords: biopower, biopolitics, panoptics, hegemony, subordinate, global health, colonial project

Mots-clés : biopouvoir, biopolitique, panoptique, hégémonie, subalterne, santé mondiale, projet colonial

\section{AUTEUR}

\section{JACQUELINE DESCARPENTRIES}

Université de Paris 8-Vincennes,

Saint-Denis. (France) 